\title{
Surface Damage and Bleaching Effect according to the Application Type of Home Tooth Bleaching Applicants
}

\author{
Na-Yeoun Tak*, Do-Seon Lim*, Hee-Jung Lim, and Im-Hee Jung ${ }^{\dagger}$ \\ Department of Dental Hygiene, The Graduate School of Health Science, Eulji University, Seongnam 13135, \\ Korea
}

Background: In this study, the bleaching effect and surface damage of two types of over-the-counter home tooth bleaching agents were explored using an in vitro study of bleaching agents applied to bovine teeth specimens for 14 days.

Methods: Domestic over-the-counter home tooth bleaching agents of gel and patch form that shared common active ingredients and manufacturers were selected and tested. The experiment specimens were made using composite resin with bovine tooth samples and then measured the initial microhardness. Specimens were then divided into a Gel group and a Patch group and underwent bleaching treatment once a day for two weeks for 30 to 60 minutes (recommended) or 7 hours. All specimens were coffee-stained prior to bleaching. The bleaching effect was measured using a spectrophotometer and surface damage was measured using a microhardness meter.

Results: The difference in color following the bleaching procedure was positive in both the Gel and Patch group, although there were no statistically significant differences in bleaching effect between groups. There was no significant difference in bleaching effect based on duration. The microhardness test revealed that both the Gel group and the Patch group had surface damage after bleaching. The greatest surface damage was found in the Patch group that had undergone a 7-hour bleaching treatment, although the differences were not statistically significant.

Conclusion: The bleaching effect of the home tooth bleaching agent was visible to the naked eye. However, longer applications than recommended did not result in greater bleaching, unlike consumers' expectations, and instead increased the chance of enamel damage. As such, there is a need for consumers to be alert and adhere to recommendations provided by each company.

Key Words: Bleaching agents, Hydrogen peroxide, Tooth bleaching

\section{Introduction}

Appearance-altering behavior to create an all-appealing, extroverted image has become one of the dominant cultures of daily life in the current generation along with the desire to enhance one's appearance ${ }^{1}$. In particular, the desire for clean, white teeth manifest in various ways, including undergoing laminate or tooth bleaching procedures ${ }^{2-4)}$.

Tooth bleaching is a procedure in which stained teeth are bleached white, which can be categorized as follows: in-office bleaching, where the individual receives treatment at a dentist's office; home bleaching, where the bleaching procedure is done at home using a personal mouthguard and expert supervision; and over-the-counter (OTC) home bleaching, where a product is purchased OTC and the bleaching procedure is done without expert supervision ${ }^{5)}$. Among these, OTC home bleaching is the most popular tooth bleaching technique as products can easily be purchased online or in stores and does not require a visit to the dentist's office for a personal mouthguard ${ }^{6}$. OTC home bleaching products include whitening toothpastes (cream type bleaching agents), patches (coated strip type 
patches applied onto the tooth surface), and gels (paint-on type bleaching agents provided in a tube or syringe $)^{7,8)}$.

Although the exact mechanism of tooth bleaching is not known for certain, it has been understood that hydrogen peroxide free radicals penetrate through enamel and dentine, causing an oxidation of the pigment inside the tooth, which results in bleaching ${ }^{9)}$. Hydrogen peroxide, the main component of bleaching agents, is a very strong oxidizing agent that is colorless and odorless. Close attention is required when using hydrogen peroxide as it may cause corrosion and chemical burns on the skin, eyes, or oral mucosa. During in-office bleaching, a high concentration ( $30 \%$ or higher) of hydrogen peroxide is often used, although there tends to be strict safety protocols in direct interventions by dentists. In contrast, home bleaching is conducted in the absence of an expert, which may put the user at risk even if low-concentration hydrogen peroxide is used ${ }^{10)}$.

In particular, whitening toothpastes are only used in short durations during the brushing process, but patches and gels can be applied repeatedly on the tooth surface for hours at a time, making them easier to abuse. In fact, side effects such as change in the enamel and hypersalivation have been reported. Kim et al. ${ }^{11)}$ have also reported that even commercially available low-concentration home bleaching agents can cause side effects such as hypersensitivity, burns, and damage to hard dental tissue if applied for longer than the recommended.

Nevertheless, most studies on tooth bleaching have been on in-office bleaching and those on home bleaching focus only on the bleaching effect or ingredients rather than the type of product, leading to limited available information for consumers aside from promotional information provided by manufacturers. Furthermore, an error rate (\%) of $71.0 \%$ $(n=233)$ was reported by Jung and Lee ${ }^{12)}$ in the question inquiring whether "more frequent tooth bleaching is beneficial for dental health" in their "knowledge and satisfaction survey on tooth bleaching', demonstrating how tooth bleaching is undertaken without accurate knowledge.

Although Yalcin and Gürgan ${ }^{13)}$ and Zantner et al. ${ }^{14)}$ have identified and compared the damage from gel- and patch-type bleaching agents, the findings from Yalcin and
Gürgan ${ }^{13)}$ had been applied to esthetic restorations, which differs slightly from tooth surface. Meanwhile, Zantner et al. ${ }^{14)}$ had used $5.9 \%$ hydrogen peroxide, which exceeds the legal recommendation of $3 \%$ in Korea, making it difficult to apply the acquired findings into the Korean context. Korean studies on bleaching patches and gels had been conducted by Park et al. ${ }^{15)}$ and Kim et al. ${ }^{16)}$. However, Park et al. ${ }^{15)}$ compared products with different components $-\mathrm{a}$ $2.5 \%$ hydrogen peroxide patch and a $10 \%$ carbamide peroxide gel. Kim et al. ${ }^{16)}$, on the other hand, demonstrated only positive bleaching effects as the authors were affiliated researchers of the manufacturer. Needless to say, risks such as surface damage were not included in the studies.

As such, this study recognizes the need for further research on Korean OTC home bleaching agents for safe and informed consumer decisions on home bleaching products. The purpose of this study, thus, is to identify the bleaching effect and the extent of enamel damage of different types of bleaching agents from a selection of geland patch-type products with identical active ingredients.

\section{Materials and Methods}

\section{Materials}

Ninety-six bovine teeth were extracted using forceps to prepare the specimens. Among these, 70 healthy teeth without caries were selected and soft tissue and stains were removed using an ultrasonic scaler.

Gel- and patch-type bleaching agents with common main active ingredients and manufacturers that differed only in type were selected among 12 Korean home bleaching products (Lumini S Gel, OTKCNT Co. Ltd, Seoul, Korea; Lumini S Patch, OTKCNT Co. Ltd; Perio $46 \mathrm{~cm}$ white now pro Gel, LG Household \& Health Care Ltd, Seoul, Korea; Perio $46 \mathrm{~cm}$ white now pro Patch, LG Household \& Health Care Ltd). In all products used in the study, $35 \%$ hydrogen peroxide solution was the main active ingredient, which contained $2.9 \sim 3.0 \%$ hydrogen peroxide. 


\section{Procedure}

\section{1) Specimen production}

Highspeed handpiece (SKU: H2050; iM3Vet Pty Ltd, Vancouver, WA, USA) and Diamond bur (TF-21, TR-11, TR-20, TF-13C, RS-11; Mani, Inc., Utsunomiya, Japan) were used to section the bovine teeth into four parts by labial (buccal), lingual and proximal surface. Each dissected specimen was trimmed to pieces $7 \times 12 \times 2 \mathrm{~mm}$ in size. Afterwards, rectangular acrylic resin (Lang Denture Resin Acrylic Self Cure Pink; Henry Schein, Inc., Melville, NY, USA) molds were prepared. Prior to curing the mold, the sectioned bovine teeth were inserted into the resin and secured. As a result, 198 specimens were produced in total.

\section{2) Microhardness pre-measurement}

The Vickers hardness number (VHN) $\left(\mathrm{kgf} / \mathrm{mm}^{2}\right)$ of all specimens prior to bleaching was measured using a microhardness meter (DMH-2; Matsuzawa Co, Tokyo, Japan). Applying a vertical load of $9.087 \mathrm{~N}$ for 10 seconds on the surface of the measurement area to create a diamond-shaped indentation, the axis of indentation was measured. In order to reduce measurement errors, two measurements were taken per specimen. Six sections (anterior labial, lingual and proximal surfaces, and posterior buccal, lingual and proximal surfaces) were included, although specimens with hardness measurements that were too high or too low were excluded ${ }^{17,18)}$. Finally, a total of 54 specimens within the average $75 \sim 85 \mathrm{VHN}$ were selected.

\section{3) Staining and grouping}

All specimens were stained by immersion in staining solution for 24 hours. Based on findings of previous research on beverages that cause extrinsic tooth discoloration that reported coffee as resulting in the greatest discoloration, sugar-free Americano (Maxim original; Kraft Foods Holdings Singapore Pte. Ltd., Seoul, Korea) was used to stain the specimen for one day in this study ${ }^{19,20)}$.

The 48 specimens were divided in half into a Gel group and a Patch group, and then the remaining six were stored separately as reference samples $(n=6 * 1)$ for bleaching effect measurements. Groups of specimens were comprised of an even mix of the six sections (anterior labial, lingual and proximal surfaces, and posterior buccal, lingual and proximal surfaces). In order to ensure consistency in the surface area to be put under bleaching treatment, only the area to be bleached was exposed and the rest of the specimen was wrapped in aluminum foil as demonstrated in Fig. 1. The bleaching surface dimensions were determined based on the smallest area after measuring the flattest and least damaged portion in specimens. The dimensions of the final application area were $4 \times 7 \mathrm{~mm}$.

\section{4) Bleaching treatment}

The bleaching treatment was performed for the recommended time (30 60 minutes) and 7 hours for each group. Here, the 7 hours was based on the average sleeping hours of Koreans as published in the 'Korea National Health and Nutrition Survey' from 2009 $2015^{21)}$. In the Gel group, $0.2 \mathrm{ml}$ of bleaching gel was applied to the tooth surface using a $1 \mathrm{ml}$ syringe (KOVAX-syringe $1 \mathrm{ml}$; Korea vaccine Co. Ltd, Seoul, Korea). In the Patch group, a bleaching patch was cut into $4 \times 7 \mathrm{~mm}$ strips and placed on the tooth surface. In order to create an environmental condition similar to that of the oral cavity, the specimen was placed on a dampened $5 \times 5$

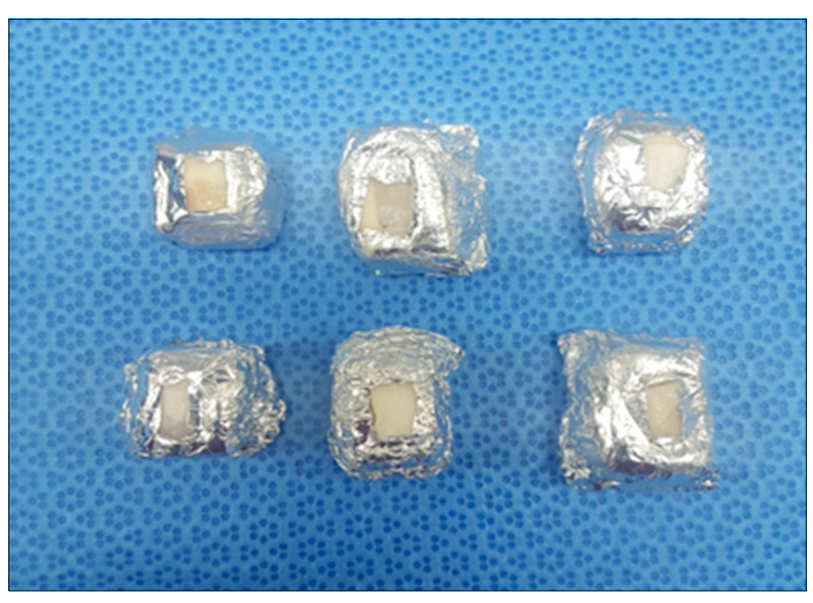

Fig. 1. Specimens exposed only the expected areas of application. Fig. 1 is a picture of the experimental specimens before whitening treatment. After coffee coloring, all the specimens surfaces were coated with foil except for the application area $(4 \times 7 \mathrm{~mm})$ so that all samples were exposed at the same size. 
mm gauze (Absorbent Gauze; Soosung Corp, Ltd., Yangsan, Korea) and stored within a $37^{\circ} \mathrm{C}$ Incubator (OF-12GW; Jeioteck Co., Daejeon, Korea). Afterwards, distilled water was used to rinse the specimens in order of application, which were then stored again in the $37^{\circ} \mathrm{C}$ Incubator until the next bleaching treatment. The bleaching treatments were carried out for 14 days as according to the recommendations provided by the manufacturer ${ }^{22)}$. That is, the bleaching treatment, rinsing and storage of specimens in the experimental groups were repeated for 14 days.

\section{5) Evaluation}

(1) Measurement of bleaching effect

After the 14 days, a spectrophotometer (DR 2700 HACH; Hach Sales \& Service Canada Ltd, Loveland, CO, USA) was used to measure the bleaching effect in each experimental group. After cleaning the tooth surface with distilled water and drying it, two measurements were taken in each specimen using the specular component included mode and standard light source D65 settings. The average value from the measurements were selected as the Commission Internationale de l'Eclairage (CIE) $\mathrm{L}^{*} \mathrm{a}^{*} \mathrm{~b}^{*}$ (CIELAB color space, 1976) value for each tooth. The CIE $L^{*} a^{*} b^{*}$ color space referred to in this study is a method of measuring color change, defined in 1976 by the $\mathrm{CIE}^{23)}$. The degree of discoloration $\left(\triangle \mathrm{E}^{*}{ }_{\mathrm{ab}}\right)$ of the specimens in the experimental group can be evaluated by comparison to the measurement values obtained for the reference samples. Values of $\triangle \mathrm{L}^{*}, \triangle \mathrm{a}^{*}, \triangle \mathrm{b}^{*}$ can be used to calculate $\triangle \mathrm{E}^{*}$ ab. $\triangle \mathrm{L}^{*}$ indicates lightness in the range of $0 \sim 100 . \triangle L^{*}=0$ defines black, while $\triangle L^{*}=100$ defines white (maximum lightness). $\triangle \mathrm{a}^{*}$ indicates green $(-)$ and red $(+)$, while $\triangle \mathrm{b}^{*}$ indicates blue $(-)$ and yellow $(+)$. The values represented in the $\mathrm{a}$ and $\mathrm{b}$ axes range between $-120 \sim+120$. The values of $\triangle \mathrm{a}^{*}=0$ and $\triangle \mathrm{b}^{*}=0$ indicate a saturation of 0 (e.g., grey) and it is understood that the greater the absolute value of $a b$, the greater the saturation. Sample specimens that had not undergone bleaching treatment were used to examine the bleaching effect on specimens in the experimental group and the color change $\left(\triangle \mathrm{E}_{\mathrm{ab}}^{*}\right)$ was converted using formula (1) below ( $\triangle \mathrm{L}^{*}$ : white-black, $\triangle \mathrm{a}^{*}$ : red-green, $\triangle \mathrm{b}^{*}$ : yellow-blue):

$$
\triangle \mathrm{E}^{*}{ }_{\mathrm{ab}}=\left(\triangle \mathrm{L}^{* 2}+\triangle \mathrm{a}^{*^{2}}+\triangle \mathrm{b}^{*^{2}}\right)^{1 / 2}
$$

(2) Measurement of microhardness

To determine the extent of tooth enamel damage, VHN measurements were taken once more using the same procedure following the bleaching treatment using a microhardness meter. The percentage microhardness loss (PML) (\%) was calculated using formula (2) below (VHN(B): initial microhardness value, VHN(A): posttreatment microhardness value):

$$
\operatorname{PML}(\%)=\{(\mathrm{VHN}(\mathrm{B})-\mathrm{VHN}(\mathrm{A})) / \mathrm{VHN}(\mathrm{B})\} \times 100
$$

\section{Analysis}

Data collected from the spectrophotometer and microhardness meter were analyzed using IBM SPSS Statistics Ver. 23.0 (IBM Corp., Armonk, NY, USA) under the 95\% significance level. The mean and standard deviation of each group were calculated to determine whether a significant difference was present in the bleaching effect and surface damage between the experimental groups. The discoloration between experimental groups was analyzed using a parametric t-test according to the normal distribution. Meanwhile, the change in microhardness was analyzed using the non-parametric Mann-Whitney test as the microhardness measurements did not follow a normal distribution pattern.

\section{Results}

\section{Comparison of bleaching effect}

All $\triangle \mathrm{E}^{*}{ }_{\mathrm{ab}}$ values were positive, indicating a significant bleaching effect. A comparison of the bleaching effects in the Gel and Patch groups that had been exposed to the bleaching agent for the recommended time revealed a $\triangle \mathrm{E}^{*}{ }_{\mathrm{ab}}$ of 23.51 for the Gel group and a $\triangle \mathrm{E}^{*}{ }_{\mathrm{ab}}$ of 19.60 for the Patch group, indicating a slightly greater bleaching effect in the Gel group, although the difference was not statistically significant ( $\mathrm{p}=0.129$, Table 1 ). When exposed to the bleaching agent for 7 hours, the value of $\triangle E^{*}$ ab for the Gel group was 23.92 and 21.12 in the Patch group, 
Table 1. Whitening Effect

\begin{tabular}{llcc}
\hline \multicolumn{1}{c}{ Time } & Type & $\triangle \mathrm{E}^{*} \mathrm{ab}^{\mathrm{a}}$ & $\mathrm{p}$-value \\
\hline \multirow{2}{*}{ Recommended time } & Gel & $23.51 \pm 4.84$ & 0.129 \\
& Patch & $19.60 \pm 7.10$ & \\
$7 \mathrm{~h}$ & Gel & $23.92 \pm 4.74$ & 0.164 \\
& Patch & $21.12 \pm 6.21$ & \\
\hline
\end{tabular}

Values are presented as mean \pm standard deviation.

${ }^{\mathrm{a}}$ The color brightness value after whitening.

where the bleaching effect in the Gel group was still greater, although not statistically significant $(\mathrm{p}=0.164$, Table 1).

\section{Comparison of surface damage}

The pre- versus post-intervention PML (\%) value were all positive, indicating a decrease in microhardness in all specimens following the bleaching treatment. The decrease of microhardness in the Gel and Patch groups that had undergone the bleaching treatment for the recommended time were $36.55 \%$ for the Gel group and $10.95 \%$ for the Patch group, indicating greater surface damage in the Gel group, although the difference was not statistically significant $(\mathrm{p}=0.644$, Table 2 ). When exposed to the bleaching agent for 7 hours, the PML of the Gel group and Patch group were $0.35 \%$ and $39.55 \%$ respectively, indicating greater surface damage in the Patch group, but this difference, too, was not statistically significant $(\mathrm{p}=0.326$, Table 2$)$.

\section{Discussion}

One's appearance is determined by a variety of factors including the presence of absence of teeth, as well as their form, arrangement and color. The aesthetics of anterior teeth, in particular, play an important role in determining an individual's first impression. As the interest in beauty is higher than ever due to recent economic prosperity, people have the tendency to wish to have whiter teeth than others in their social group ${ }^{24)}$.

Following the release of the Nightguard home bleaching technique using 10\% carbamide peroxide in 1989 by Haywood and Heymann ${ }^{25}$, home bleaching out of the dentist's office became commercialized. Recently, with
Table 2. Surface Damage

\begin{tabular}{clcc}
\hline \multicolumn{1}{c}{ Time } & Type & PML $^{\mathrm{a}}(\%)$ & p-value \\
\hline Recommended time & Gel & $36.55 \pm 21.99$ & 0.644 \\
& Patch & $10.95 \pm 71.37$ & \\
$7 \mathrm{~h}$ & Gel & $0.35 \pm 72.90$ & 0.326 \\
& Patch & $39.55 \pm 120.66$ & \\
\hline
\end{tabular}

Values are presented as mean \pm standard deviation.

PML: percentage microhardness loss.

${ }^{\mathrm{a}}$ Microhardness damage value after whitening.

development of more convenient OTC home bleaching products that are more consumer-friendly, the demand for home bleaching has further increased. Nonetheless, as mentioned in the Introduction section, research on the safety of tooth bleaching have mostly focused only on in-office bleaching using high-concentration hydrogen peroxide, with insufficient research on home bleaching techniques that use relatively lower concentrations of hydrogen peroxide. OTC home bleaching agents, especially, are increasingly diverse in form, leaving most consumers to rely only on information provided by manufacturers in distinguishing between the different products. Consequently, accounts of adverse events from home bleaching from consumers are also increasing ${ }^{6}$. As such, this study aimed to provide basic data on home bleaching products by comparing the bleaching effect and surface damage attributed to Patch- and Gel-type bleaching agents, among commercially available home bleaching products.

First, the CIE $\mathrm{L}^{*} \mathrm{a}^{*} \mathrm{~b}^{*}$ values and color change $\left(\triangle \mathrm{E}_{\mathrm{ab}}{ }\right)$ were calculated in order to assess tooth bleaching effects. Gross and Moser $^{26)}$ established that a $\triangle \mathrm{E}^{*}{ }_{\mathrm{ab}}$ value between $0 \sim 2$ indicates a color change that is invisible to the naked eye, a value between $2 \sim 3$ indicates a color change just visible to the naked eye, a color change value within the range of $3 \sim 8$ is distinguishable by the naked eye, and a value above 8 is apparent. Ruyter et al. ${ }^{27)}$ also established that since a color change over 3.3 was distinguishable to the naked eye, a color change below 3.3 would not be clinically significant. According to the findings of this study, the average color change was sufficiently distinguishable at $\triangle \mathrm{E}^{*}{ }_{\mathrm{ab}}=22.12$. Similar findings were reported in a previous study by Bahng et al. ${ }^{24)}$ where a color change of 18.8 was observed in a group treated 
with a Gel-type bleaching agent containing 3.0\% hydrogen peroxide. The reason why the color change values were greater than the standards put forth by Gross and Moser $^{26}$ in the two studies may be attributed to the fact that the specimen used had undergone an artificial tooth surface staining process. For instance, in a previous study by Kim et al. ${ }^{11)}$, where the bleaching process took place without previous staining, the average color change was $\triangle E^{*}{ }_{a b}=$ 3.40 , but in the case of another study by Kim et al. ${ }^{16)}$ that used specimens that had undergone a staining process, a greater color change of $\triangle \mathrm{E}^{*}{ }_{\mathrm{ab}}=34.02$ was detected.

In terms of bleaching effect by the time exposed to the bleaching agent, it has typically been understood that longer exposure led to a greater bleaching effect. Previous research from Monaghan et al. ${ }^{28)}$ also demonstrated, following a 2-week experiment, that bleaching effect was significantly greater with longer exposure. However, in this study, the difference in $\triangle \mathrm{E}^{*}$ ab values of the specimens exposed for the recommended time and those exposed for 7 hours were 0.41 for the Gel-type and 1.52 for the Patch-type, indicating a color change within the $0 \sim 2$ range, indicating that the color change was invisible to the naked eye. In other words, there was no additional bleaching effect with longer exposure time, which contradicted the aforementioned statement that longer exposure resulted in greater bleaching effect. Such differences may be attributed to the different safety management standards of domestic versus foreign home bleaching agents. The home bleaching product used by Monaghan et al. ${ }^{28)}$, for example, demonstrated additional bleaching effects with additional exposure as it contained over $10 \%$ hydrogen peroxide, whereas the home bleaching product used in this study contained $2.9 \sim 3 \%$ hydrogen peroxide (35\% hydrogen peroxide solution), which may explain why no additional bleaching was observed with longer exposure. As the OTC tooth bleaching products sold in Korea cannot exceed a relatively low $3 \%$ hydrogen peroxide concentration, it is not to be expected that an extended duration of treatment would result in greater bleaching effects.

In terms of bleaching effect based on application type, a previous study by Kim et al. ${ }^{16)}$ demonstrated that the Patch-type had an approximately $27 \%$ greater bleaching effect than the Gel-type. It was also reported that such results could be attributed to the fact that the Patch-type was more thoroughly adhered to the tooth surface, delivering the bleaching agent more effectively, in one direction. However, the results obtained in this study contradicted previous study findings. The bleaching effect observed in the Gel-types was higher in both the group exposed to the bleaching agent for the recommended time, as well as the group exposed for 7 hours, not to mention that the difference was not statistically significant. For this, additional research with a longer treatment and experimental duration will be needed in the future.

Although the exact mechanism of tooth bleaching has not yet been known, it has been understood that hydrogen peroxide, its main active ingredient, is broken down inside the oral cavity to form active oxygen, which then penetrates into the teeth and reacts with the substances that caused discoloration, thus resulting in a bleaching effect $^{29)}$. During this process, pores are formed on the surface of the enamel due to demineralization, which results in irregular prisms that reduce resistance towards caries and abrasion ${ }^{30)}$.

Microhardness tests can be used as a method to measure surface damage. In this study, the microhardness level (VHN) was measured to identify the degree of surface damage (PML), which demonstrated an approximately $10 \sim 36 \%$ decrease in microhardness upon recommended exposure to bleaching treatment. Similarly, in a study by Jung and Lee ${ }^{31)}$, who measured the pre- and post-treatment microhardness (VHN) by the same method, microhardness scores of 250.7 PML pre-treatment and 142.5 PML post-treatment were reported, demonstrating an approximately $43.15 \%$ decrease in microhardness. Here, the higher surface damage observed by Jung and Lee ${ }^{31)}$ can be attributed to the longer exposure of the specimens to the bleaching agents. In this study, a 35\% hydrogen peroxide solution was used for 2 weeks, while Jung and Lee ${ }^{31)}$ used a $15 \%$ hydrogen peroxide solution for 4 weeks, which resulted in greater surface damage despite using a lower concentration hydrogen peroxide solution. In a study by Lee et al. ${ }^{32)}$ who used a bleaching agent of the same hydrogen peroxide solution concentration as used in this study (35\%) for bleaching treatments 30 minutes, 1 hour 
and 2 hours in duration for 1- and 2-week periods each, an increasingly significant decrease in microhardness was reported, reinforcing the occurrence of greater surface damage with longer exposure. As such, most previous research reported a decrease in surface microhardness following bleaching treatment despite differences in microhardness scores based on duration of exposure and bleaching agent used, supporting the results of this study ${ }^{33)}$. Nevertheless, some previous research demonstrated an increase in microhardness following bleaching treatment ${ }^{14,34)}$. Such results, however, seem to have derived from factors such as using a bleaching agent containing fluorine or storing the specimen in artificial saliva. It is presumed that fluorine and the remineralization components in saliva inhibited the demineralizing effect of the bleaching agent. In fact, remineralization was observed by both Zantner et al. ${ }^{14)}$, who reported a $12 \sim 40 \%$ surface damage following bleaching treatment but stored the damaged specimen in artificial saliva for 6 weeks, and Attin et al. ${ }^{35}$, who used a bleaching agent containing fluorine. Such findings may imply the need to use fluorine-containing bleaching agents or follow up a bleaching treatment with a fluorine treatment in the future.

With regards to surface damage in Gel- and Patch-type bleaching products, Yalcin and Gürgan ${ }^{13)}$ reported greater surface damage from Patch-type bleaching products, although in this study, the difference in surface damage between the Gel and Patch groups were not statistically significant. Nonetheless, the surface damage in the Gel group was higher upon recommended exposure, while the surface damage in the Patch group was higher upon 7 hours of exposure. Overall, the Patch group exposed to bleaching treatment for 7 hours demonstrated the greatest decrease in microhardness across all experimental groups. In a previous study by Zantner et al. ${ }^{14)}$, as well, a greater reduction in microhardness was observed in the use of Patch-type bleaching products, based on the pre- and post-treatment difference in Knoop Hardness Number of 63.4 and 92.2 in the Gel group and Patch group, respectively. Such results can be attributed to the difference in the duration of action of each application type. For instance, Gel-type products have an advantage of coating uneven tooth surfaces with their liquid consistency unlike their Patch-type counterparts, but quickly become inactive as the bleaching agent is constantly in contact with saliva ${ }^{16}$. On the other hand, Patch-type bleaching agents, comprised of a thin, flexible polyethylene film that is coated on one side with a bleaching agent with a low moisture content, can be adhered securely to the tooth surface with the other side of the film acting as a protective barrier against saliva to prevent the dilution of the bleaching agent ${ }^{16}$. It is presumed that the Patch-type products used in previous studies also demonstrated significantly greater surface damage than Gel-type products for the same reasons, whereas a statistically significant difference was not observed in this study due to its use of in vitro experiments where the condition of the oral cavity could not be perfectly reproduced. Despite this, greater surface damage was observed in the Patch-type bleaching products in this study as well, as the patches remained adhered to the specimen for 7 hours in spite of the $37^{\circ} \mathrm{C}, 100 \%$ humid incubator condition. Therefore, further studies complementing the limitations of an in vitro study using in vivo or in situ study designs may be needed in the future to evaluate the safety of bleaching agents.

In conclusion, it is evident from the results of this study that there is no greater bleaching effect with prolonged treatment past the recommended duration as expected by consumers, but rather an increased potential for enamel damage. Mor et al. ${ }^{36)}$ reported that the surface microdamage caused by hydrogen peroxide could significantly increase the surface adhesion of bacteria, while Goldberg et al. ${ }^{37)}$ reported that such surface damage could cause additional exogenous staining, increasing the possibility of discoloration. Therefore, there is a need for manufacturers to convey not only the cosmetic function of the products, but also the risks involved with their use, and for consumers to be cautious and follow the manufacturers' recommendations. Additionally, as the Patch-type did not have a greater bleaching effect compared to the Gel-type, but rather a risk of additional surface microdamage with prolonged use due to its adhesive property, there is a need for Patch-type bleaching products to be removed promptly after the recommended duration. In conclusion, this study 
explored the bleaching effect and safety of bleaching products by type and may be used as basic data to inform consumers' choices of home bleaching products.

\section{Notes}

\section{Conflict of interest}

No potential conflict of interest relevant to this article was reported.

\section{Ethical approval}

This article does not require an IRB because it used tissue that was thrown away from the carcasses of animals.

\section{Author contributions}

Conceptualization: Na-Yeoun Tak. Data acquisition: Na-Yeoun Tak. Formal analysis: Na-Yeoun Tak and Do-Seon Lim. Funding: None. Supervision: Im-Hee Jung. Writing-original draft: Na-Yeoun Tak and Do-Seon Lim. Writing - review \& editing: Na-Yeoun Tak, Im-Hee Jung, Do-Seon Lim, and Hee-Jung Lim.

\section{ORCID}

Na-Yeoun Tak, https://orcid.org/0000-0001-9901-9411

Do-Seon Lim, https://orcid.org/0000-0003-4602-3323

Hee-Jung Lim, https://orcid.org/0000-0002-4738-3032

Im-Hee Jung, https://orcid.org/0000-0002-8645-1587

\section{References}

1. Park SH, Park HJ: A comparative study of beauty involvement by age groups according to conspicuous consumption tendency. J Korea Soc Beauty Art 12: 67-81, 2011.

2. Samorodnitzky-Naveh GR, Geiger SB, Levin L: Patients' satisfaction with dental esthetics. J Am Dent Assoc 138: 805-808, 2007.

https://doi.org/10.14219/jada.archive.2007.0269

3. Gerlach RW: Shifting paradigms in whitening: introduction of a novel system for vital tooth bleaching. Compend Contin Educ Dent (29): S4-S9, 2000.

4. Sim YW, Jung SH: Effect of fluoridated bleaching agents and post-treatment fluoride application on the color and microhardness of enamel surface. J Dent Hyg Sci 10: 295300, 2010.

5. Heymann HO: Tooth whitening: facts and fallacies. Br Dent J 198: 514, 2005. https://doi.org/10.1038/sj.bdj.4812298

6. Goo HJ, Kwun HS, Park JH, et al.: Tooth color reduction and consequent patient satisfaction after office and home bleaching: a 6-month follow-up study. J Korean Acad Oral Health 39: 3-8, 2015. https://doi.org/10.11149/jkaoh.2015.39.1.3

7. Mount GJ, Hume WR, Ngo HC, Wolff MS: Preservation and restoration of tooth structure. 3rd ed. Wiley-Blackwell, Hoboken, pp.185-194, 2016.

8. McGuckin RS, Babin JF, Meyer BJ: Alterations in human enamel surface morphology following vital bleaching. J Prosthet Dent 68: 754-760, 1992. https://doi.org/10.1016/0022-3913(92)90197-i

9. Nathoo SA: The chemistry and mechanisms of extrinsic and intrinsic discoloration. J Am Dent Assoc 128 Suppl: 6S-10S, 1997.

https://doi.org/10.14219/jada.archive.1997.0428

10. Lee JK, Min SH, Hong ST, et al.: Clinical study of shade improvement and safety of polymer-based pen type BlancTic Forte whitening agent containing $8.3 \%$ Carbamide peroxide. J Korean Acad Conserv Dent 34: 154-161, 2009. https://doi.org/10.5395/JKACD.2009.34.2.154

11. Kim SY, Ahn JH, Kim JY, Kim JW, Park SH, Cho KM: Efficacy of a self -applied paint - on whitening gel combined with wrap. J Dent Rehabil Appl Sci 34: 175-185, 2018. https://doi.org/10.14368/jdras.2018.34.3.175

12. Jeong YR, Lee JY: Knowledge and satisfaction status of tooth bleaching. J Korea Contents Assoc 18: 403-414, 2018. https://doi.org/10.5392/JKCA.2018.18.10.403

13. Yalcin F, Gürgan S: Effect of two different bleaching regimens on the gloss of tooth colored restorative materials. Dent Mater 21: 464-468, 2004. https://doi.org/10.1016/j.dental.2004.07.011

14. Zantner C, Beheim-Schwarzbach N, Neumann K, Kielbassa AM: Surface microhardness of enamel after different home bleaching procedures. Dent Mater 23: 243-250, 2007. https://doi.org/10.1016/j.dental.2006.06.044

15. Park YW, Park SH, Kim JW, Cho KM: The effects of tooth bleaching agents on microhardness of enamel in situ. $\mathbf{J}$ 
Korean Acad Conserv Dent 31: 470-476, 2006.

https://doi.org/10.5395/JKACD.2006.31.6.470

16. Kim JH, Bang SE, Kim JY, Ahn JH: Comparison of tooth whitening efficacy between gel and strip with light activator. J Dent Hyg Sci 17: 481-486, 2017. https://doi.org/10.17135/jdhs.2017.17.6.481

17. Woo HS, Shim YS: Effect of $15 \%$ carbamide peroxide agents with and without potassium nitrate and fluoride(PF) on the tooth color and surface microhardness of human enamel. $\mathrm{J}$ Korea Contents Assoc 12: 353-360, 2012. https://doi.org/10.5392/JKCA.2012.12.01.353

18. Cho MJ, Ha MO, Oh HN: The preventive effect of fluoride materials on the dental caries by dental polishing prior to fluoride application. J Korean Soc Dent Hyg 12: 113-122, 2012. https://doi.org/10.13065/JKSDH.2012.12.1.113

19. Kim EH, Lee DH, Oh HS: Effects of the repetitive tasting of different blending types of coffee on teeth stain during home bleaching. J Korean Soc Dent Hyg 10: 955-963, 2010.

20. Kim SH, Choi HS, Roh JY, Kim KM: The effect of $15 \%$ carbamide peroxide on the surface roughness and staining of esthetic restoratives. J Dent Hyg Sci 13: 165-173, 2013.

21. Shin DY, Hur J, Cho KH, Cho EH: Trends of self-reported sleep duration in Korean adults: results from the Korea National Health and Nutrition Examination Survey 2007-2015. Sleep Med 52: 103-106, 2018. https://doi.org/10.1016/j.sleep.2018.08.008

22. Shin HE, Im SU, Kim EK, et al.: Effects of a whitening strip combined with a desensitizing primer on tooth color. J Korean Acad Oral Health 40: 31-37, 2016. https://doi.org/10.11149/jkaoh.2016.40.1.31

23. Seghi RR, Johnston WM, O'Brien WJ: Spectrophotometric analysis of color differences between porcelain systems. J Prosthet Dent 56: 35-40, 1986. https://doi.org/10.1016/0022-3913(86)90279-9

24. Bahng K, Yoo S, Park Y: Maintenance of teeth brightness with $0.3 \% \mathrm{H}_{2} \mathrm{O}_{2}$ containing toothpaste after use of $3.0 \%$ $\mathrm{H} 2 \mathrm{O} 2$ brightening gel. J Korean Acad Dent Health 31: 167-175, 2007.

25. Haywood VB, Heymann HO: Nightguard vital bleaching. Quintessence Int 20: 173-176, 1989.

26. Gross MD, Moser JB: A colorimetric study of coffee and tea staining of four composite resins. J Oral Rehabil 4: 311-322, 1977. https://doi.org/10.1111/j.1365-2842.1977.tb00997.x

27. Ruyter IE, Nilner K, Möller B: Color stability of dental composite resin materials for crown and bridge veneers. Dent Mater 3: 246-251, 1987. https://doi.org/10.1016/S0109-5641(87)80081-7

28. Monaghan $\mathrm{P}$, Trowbridge $\mathrm{T}$, Lautenschlager E: Composite resin color change after vital tooth bleaching. J Prosthet Dent 67: 778-781, 1992. https://doi.org/10.1016/0022-3913(92)90581-t

29. Alqahtani MQ: Tooth-bleaching procedures and their controversial effects: a literature review. Saudi Dent J 26: 33-46, 2014. https://doi.org/10.1016/j.sdentj.2014.02.002

30. Bitter N, Sanders J: Electron microscopy study of four bleaching agents on enamel surfaces. J Dent Res 71(1_suppl): 600-602, 1992.

31. Jung IS, Lee HJ: Comparision of change on the enamel of tooth after bleaching. J Korean Soc Oral Health Sci 2: 64-70, 2014.

32. Lee HJ, Kim MY, Kim KH, Kwon TY: 35\% Hydrogen peroxide gel in the whitening effect and enamel changes. $\mathrm{J}$ Dent Hyg Sci 8: 255-260, 2008.

33. Jeoung MA, Oh HS, Shim YS: Effect of 35\% hydrogen peroxide with dicalcium phosphate dihydrate on the tooth whitening and microhardness. J Korea Contents Assoc 10: 235-242, 2010. https://doi.org/10.5392/JKCA.2010.10.11.235

34. Attin T, Kielbassa AM, Schwanenberg M, Hellwig E: Effect of fluoride treatment on remineralization of bleached enamel. J Oral Rehabil 24: 282-286, 1997.

35. Attin T, Kocabiyik M, Buchalla W, Hannig C, Becker K: Susceptibility of enamel surfaces to demineralization after application of fluoridated carbamide peroxide gels. Caries Res 37: 93-99, 2003. https://doi.org/10.1159/000069015

36. Mor C, Steinberg D, Dogan H, Rotstein I: Bacterial adherence to bleached surfaces of composite resin in vitro. Oral Surg Oral Med Oral Pathol Oral Radiol Endod 86: 582-586, 1998. https://doi.org/10.1016/s1079-2104(98)90350-x

37. Goldberg M, Claisse-rinquette A, Bourd-Boittin K, Le Denmat D: Tooth bleaching treatment. J Am Dent Assoc 10-46, 2005. 